Service social

\title{
La vie associative des personnes âgées
}

\section{Suzanne Moffet}

Volume 34, numéro 1, 1985

Personnes âgées, milieux de vie et pratiques sociales

URI : https://id.erudit.org/iderudit/706251ar

DOI : https://doi.org/10.7202/706251ar

Aller au sommaire du numéro

Éditeur(s)

École de service social de l'Université Laval

ISSN

1708-1734 (numérique)

Découvrir la revue

Citer cet article

Moffet, S. (1985). La vie associative des personnes âgées. Service social, 34(1),

55-76. https://doi.org/10.7202/706251ar d'utilisation que vous pouvez consulter en ligne.

https://apropos.erudit.org/fr/usagers/politique-dutilisation/ 
Moffet, Suzanne, conseillère en planification et programmation, Conseil régional de la santé et des services sociaux, région de Québec.

\section{La vie associative des personnes âgées}

\section{Suzanne Moffet}

Le capitalisme industriel a connu l'émergence d'un nouveau statut pour les personnes âgées: celui de retraité. ${ }^{1}$ Aujourd'hui, dans une société dite post-industrielle, être retraité ou pré-retraité signifie qu'à un certain âge, par exemple 55, 60 ou 65 ans, la personne se retrouve officiellement "au repos» après avoir assumé divers rôles liés au travail ou à l'éducation familiale. Elle doit alors faire face à 10, 20 ou, pour être optimiste, 30 ans de vie marqués par le déclin physiologique mais également par une liberté d'être.

L'accroissement de l'espérance de vie, l'importance démographique grandissante de ce groupe particulier et l'institutionnalisation des pensions de vieillesse ont contribué à créer une situation historique tout à fait nouvelle. Celle-ci engendre, depuis les années 1950, toute une série de préoccupations et de réponses sociales. C'est ainsi qu'a pris naissance la gérontologie, comme discipline, et, dans le champ des sciences médicales, de la psychologie et de l'intervention sociale, des spécialisations ont émergé pour répondre aux besoins de cette nouvelle clientèle. Le milieu de vie s'est progressivement réajusté pour tenir compte de l'accroissement de cette population, par exemple par la construction de résidences spécialisées et même de villages de retraités, ou encore par l'introduction de cette nouvelle clientèle cible dans le commerce. En résumé, la vieillesse devient l'objet de politiques sociales pour l'État.

Parmi les multiples réajustements de l'organisation sociale pour tenir compte du vieillissement, on observe un phénomène significatif : l'apparition d'associations volontaires spécifiques qui, depuis quelques années, connaissent un essor considérable dans toutes les sociétés modernes. On en distingue déjà deux grandes catégories: les unes regroupent majoritairement des personnes âgées, et les autres, vouées 
au bien-être de celles-ci, sont organisées et composées en grande partie par des professionnels.

Notre propos, ici, consistera à examiner la nature et la portée des associations de personnes âgées afin de mieux comprendre la signification de ce phénomène eu égard au devenir de la vieillesse dans la société moderne.

\section{La vie associative, une couleur de notre temps}

Depuis 1950, le développement associatif a fait des progrès remarquables, et il s'enracine même dans toutes les cultures occidentales. Les associations volontaires deviennent ainsi de plus en plus l'objet de recherches scientifiques. Même les journaux font état de "l'associationnite». Par ailleurs, une encyclopédie ${ }^{2}$ regroupant les diverses associations américaines est publiée depuis 1959 et cet inventaire s'accroît d'année en année.

Le besoin de regroupement est intense et tous les motifs peuvent y servir de prétextes, qu'ils soient fort sérieux et entraînent la création d'association professionnelle comme, par exemple, une corporation des travailleurs sociaux, ou que les motifs soient inusités tel un intérêt marqué pour le dindon (les Amis du dindon).

On observe que les changements structurels conséquents à l'urbanisation et à l'industrialisation ont favorisé le développement d'associations volontaires. Maintenant, la nouvelle famille est réduite à son minimum ; on observe aussi l'augmentation de la monoparentalité. Les relations soutenues avec la famille étendue ont changé et elles ne sont plus maintenues de façon systématique comme avant. Par ailleurs, les relations de voisinage sont souvent inexistantes. La participation associative constitue, dans ce contexte, un mode de relation et d'expression librement choisi qui permet de reconstituer des solidarités sur la base d'affinités personnelles ou d'intérêts spécifiques. Outre les besoins d'ordre relationnel et affectif, les associations volontaires comblent également, selon les spécificités propres à divers milieux, d'autres besoins, qu'ils soient d'ordre politique, religieux, professionnel, éducatif ou récréatif. Elles sont multiples et variées et rejoignent toutes les strates de la population.

Pour Dion, "l'association volontaire est l'union officielle, durable, sans but lucratif de plusieurs personnes qui se sont entendues de leur plein gré pour mettre en commun certaines ressources et poursuivre ensemble de façon régulière et par leurs propres moyens des fins 
particulières qui leur sont tangentielles. ${ }^{3} \mathrm{Et}$, comme le faisait observer Meister, ${ }^{4}$ elles peuvent être plus ou moins perméables à l'influence de la société globale. Plusieurs typologies ont été proposées par les chercheurs ${ }^{5}$ pour les caractériser. On a ainsi tenté de les classifier à partir notamment de leur taille, du type d'adhérents, du genre d'activités, des objectifs poursuivis et des fonctions assumées, du degré de solidarité interne ou encore du degré de conflit avec l'environnement social. Elles peuvent également être distinguées à partir de leur position dans l'axe expressif/instrumental, facteur qui reflète les intérêts marqués d'un groupe pour le développement personnel de ses membres (expressif) ou pour réaliser des objectifs de changement social ou d'action communautaire (instrumental). Cette distinction expressive/instrumentale, fréquemment utilisée, permet de caractériser les fonctions principales d'un groupe et d'en apprécier les tendances selon une perspective diachronique.

Selon le président de Centraide Montréal, il appert, pour plusieurs, "qu'une société ne peut être vivante sans cette multitude de groupes bénévoles et communautaires, capables de prendre en main leurs propres réalités et leur vécu quotidien ". ${ }^{6}$ L'importance des associations volontaires pour l'entraide et le développement communautaire a été maintes fois soulignée; mais, dans un contexte où les limites de l'ÉtatProvidence apparaissent de plus en plus évidentes, cette fonction instrumentale revêt une signification particulière.

Bien que les associations spécifiques de personnes âgées soient inscrites, de façon certaine, dans la vie sociale depuis les années 1960, il s'est fait relativement peu de recherches dans ce domaine. Pourtant, ces groupes s'accroissent, leurs fonctions se diversifient et les adhérents augmentent. Pour cette population, la vie associative représente un effort pour développer des formes de solidarité et d'expression affective mieux adaptées aux exigences d'une société moderne, répondre à des aspirations nouvelles mais aussi à des problèmes spécifiques. Ces manifestations sont particulièrement importantes si l'on considère le "cortège de misères" qui vient avec le vieillissement: pauvreté, maladies, isolement, solitude, usure psychologique, préjugés, sentiment d'inutilité.

Leur vie associative s'exprime à travers diverses formes, qui vont des clubs locaux aux organismes nationaux. Un regard sur ces regroupements, tels qu'ils ont été présentés dans les publications américaines, françaises et québécoises, nous permettra d'en explorer les contours mais aussi de mieux comprendre la signification de ce phénomène dans le contexte du vieillissement des populations. 


\section{La vie associative des personnes âgées : manifestations et questionnements}

\section{États-Unis}

\section{a) Le mouvement Townsend}

C'est autour de l'enjeu des pensions de vieillesse que le mouvement Townsend a pris naissance en 1932 à titre de première manifestation associative à grande échelle des personnes âgées. Amorcé aux États-Unis et connu originalement sous le nom de Old Age and Revolving Pensions Ltd., il réussit, sous la gouverne de son leader charismatique Francis $\mathrm{E}$. Townsend, à mobiliser une bonne proportion de personnes âgées à la grandeur du pays dans le but de faire voter une législation pour que les individus de 60 ans et plus aient droit à une pension mensuelle de $\$ 200$, à la condition de renoncer à un emploi rémunéré. Des clubs furent mis sur pied et des cotisations individuelles furent prélevées pour appuyer ces revendications.

Plus qu'un plan de pension, cette proposition législative avait comme objectif de donner un pouvoir d'achat aux masses de personnes âgées lourdement défavorisées pendant la grande Dépression des années 1930. L'objectif était cependant fort irréaliste, compte tenu du montant de pension demandé et de la charge fiscale démesurée que devrait alors imposer l'État pour financer un tel plan à une époque où ce dernier n'était pas interventionniste. Dans une société industrielle fondée sur une mentalité de prévoyance individuelle, l'introduction d'une responsabilité de l'État allait à l'encontre des valeurs capitalistes véhiculées par les leaders politiques. Un tel contexte socio-politique, jumelé aux difficultés internes du mouvement (escroquerie, faiblesse organisationnelle, rivalités), firent avorter le projet collectif du docteur Townsend et le mouvement s'effrita après 1936. Néanmoins, cette expérience eut comme effet de sensibiliser l'opinion publique aux problèmes des personnes vieillissantes, d'amener une prise de conscience de leur pouvoir de mobilisation et d'introduire l'idée d'une pension de vieillesse dans la société américaine. Selon Carlie, ${ }^{7}$ cette manifestation collective doit être perçue comme un événement isolé qui émane d'une conjoncture socio-économique de crise particulièrement favorable à la revendication populaire. Pratt ${ }^{8}$ l'interprète toutefois comme le début d'un mouvement socio-politique de personnes âgées qui trouve son fondement dans l'éveil d'une prise de conscience de groupe, à savoir une misère qui appelle des solutions collectives. 
En France et au Canada, il n'y a pas eu, à cette époque, d'agitation d'une telle ampleur au sein de la population âgée. II faut attendre l'après-guerre pour que se dessine résolument dans ces pays les débuts d'une vie associative spécifique au même groupe.

De 1935 à 1960, aux États-Unis, ces regroupements sont peu visibles sur la scène politique. Cependant, on observe, sur une base locale, l'émergence de diverses structures telles: les groupes sociaux, les Senior Clubs, les associations en fonction de champs d'intérêts divers.

\section{b) La structure américaine}

1. Les Senior Clubs. À New York, dans les années 1940, sont apparus les premiers Senior Clubs, dont la mise sur pied fut encouragée par les gouvernements et les agences de bien-être. Les objectifs de ces centres étaient très diversifiés bien que le loisir, l'entraide et la socialisation figuraient parmi les préoccupations principales. Contrairement au mouvement Townsend, l'activisme politique en était pratiquement absent.

En 1960, environ $2 \%$ de la population âgée fréquente les Senior Clubs. Pratt ${ }^{9}$ souligne que ces clubs impressionnaient la population en général en raison de l'implication marquée et de la cohésion des groupes; elle ne s'attendait probablement pas à ce que de tels regroupements deviennent opérationnels. Une telle attitude, bien que paternaliste, confirme cependant la capacité réelle de cette forme de vie associative pour répondre aux besoins des personnes âgées, et pour s'inscrire de façon permanente dans la structure de la vie sociale. Quinze ans plus tard, en 1975, une enquête du National Council of the Aging, ${ }^{10}$ confirmait que la clientèle des Senior Clubs avait presque décuplée, pour atteindre $18 \%$ du nombre total des 65 ans et plus. La plupart de ceux-ci ont élargi leurs fonctions au profit d'actions communautaires nouvelles liées aux problèmes d'argent ou de santé et à l'habitat. Le club devient ainsi "un pôle d'organisation sociale totale à partir d'une base de loisirs". ${ }^{11}$

2. Initiatives religieuses et professionnelles. Parallèlement au développement des Senior Clubs, divers groupes religieux organisèrent, au niveau local, des services pour les personnes âgées et favorisèrent la. mise sur pied de centres de jour, groupes récréatifs et bénévoles. De même, les syndicats et associations professionnelles créèrent des regroupements spécifiques aux retraités issus de leurs milieux; plans de retraite, cours de préparation à la retraite, rencontres éducatives figurent parmi la liste des réalisations par et pour les retraités. 
3. Les Associations nationales de personnes âgées. Depuis les années 1960, des associations nationales de personnes âgées émergent sur la scène politique et sociale. Les Gray Panthers, le National Council of Senior Citizens (N.C.S.C.) et l'American Association of Retired Persons (A.A.R.P.) représentent actuellement les regroupements les plus larges en termes d'adhérents ainsi que les plus visibles socialement. On en retrouve aussi plusieurs composés de retraités de secteurs occupationnels tels le National Retired Teachers Association et le National Association of Retired Federal Employees. Les minorités noires, espagnoles et indiennes ont aussi des représentations par le biais du National Council on the Black Aged, le National Association for Spanish Speaking Elderly et le National Indian Council on the Aging.

\section{c) Un nouveau pouvoir gris?}

Lors d'une conférence universitaire sur le vieillissement, Rose ${ }^{12}$ soulignait l'accroissement du nombre d'associations volontaires pour les personnes âgées dont certaines, tout en maintenant des fonctions sociales et récréatives, ${ }^{12}$ agissaient aussi en tant que groupes de pression dans le but de faire passer des lois pour améliorer leurs conditions de vie. Cet auteur voyait, à travers ces manifestations, les signes de l'émergence d'une conscience collective politique au sein de cette population.

Pratt ${ }^{13}$ s'est par ailleurs interrogé sur le rôle des associations nationales dans l'orientation des politiques américaines en faveur de la population âgée. Il a notamment examiné la façon dont ces groupes se sont taillés un statut politique. À partir des années 1970, des associations auraient, selon lui, atteint un niveau de maturité les habilitant à mieux intervenir sur le plan de l'action politique pour défendre leurs intérêts. Cette maturité s'illustre de plusieurs façons. Elles sont mieux organisées qu'avant, plus efficaces dans l'art de la communication de leur intérêt et, également, mieux nanties financièrement. De plus, elles ont acquis, comme porte-parole officiel, une place légitime face aux pouvoirs publics. Pratt leur attribue également un rôle significatif dans l'émergence de la prise de conscience collective des problèmes de la vieillesse. Il estime que le gray lobby est nécessaire pour s'assurer que les décideurs politiques soient attentifs et réagissent aux besoins des personnes âgées. Cependant, d'autres analystes du rôle politique des associations comme Hartoyan ${ }^{14}$ soulignent, qu'en général, leurs réalisations demeurent peu impressionnantes comparativement à celles d'autres groupes d'intérêt. Mais, compte tenu du poids démographique 
grandissant des personnes âgées, il faut inévitablement s'attendre à un renforcement de leur pouvoir sur les décisions politiques en leur faveur.

Dans la réalisation de leurs objectifs, les associations de personnes âgées sont confrontées, comme pour plusieurs groupes de pression, à de nombreux problèmes, notamment aux limites structurelles du système politique et aux rapports de classe. Mais, de façon plus spécifique, elles se heurtent aux contraintes inhérentes à l'organisation de la vie associative de personnes vieillissantes. Ces deux derniers problèmes apparaissent particulièrement importants ; ceci justifie donc qu'on les examine de plus près.

\section{d) Vie associative et rapports de classe}

Les associations volontaires, selon Levasseur "participent [...] aux mécanismes de la distinction sociale, de l'accès inégal à la production et à la consommation socio-culturelle». ${ }^{15}$ Les groupes de personnes âgées ne sont pas exempts de cette dynamique, comme le révèle également un article récent de Pratt. ${ }^{16}$ Comparant les types d'enjeux politiques et le membership de trois grandes associations nationales, il met en évidence le rapport entre leurs aspirations de classe et leurs luttes socio-politiques. Il fait particulièrement référence aux Gray Panthers, à l'American Association of Retired Persons et au National Council of Senior Citizens. La constitution sociale différenciée de ces groupes se réflète dans leur philosophie d'action et dans leur définition des besoins.

L'appartenance selon la classe sociale transparaît clairement dans les débats américains sur la retraite et sur la sécurité sociale. L'A.A.R.P., constituée principalement de personnes âgées des classes moyenne et supérieure, s'est farouchement levée contre la retraite obligatoire et en a même fait son objectif principal. Pour ce groupe, le travail est un droit et permet de se réaliser.

Par contre, le N.C.S.C. bien qu'étant, en principe, favorable à l'abolition de l'âge chronologique comme facteur discriminant afin d'établir la prise de retraite, ne voyait pas cet enjeu comme prioritaire. Regroupant surtout des retraités de classe ouvrière (cols bleus) et de minorités ethniques, dont les conditions de travail sont souvent fort difficiles, la retraite et même la pré-retraite représentent, à plusieurs égards, la libération d'un travail aliénant et souvent nuisible pour la santé. Par ailleurs, la question des revenus et, en particulier les bénéfices de la sécurité sociale, constituent pour ce même groupe un enjeu beaucoup plus vital. Conséquemment, le N.C.S.C. s'est 
impliqué activement, par exemple, dans la campagne pour l'adoption des amendements à la sécurité sociale, en 1972, qui contribuèrent à bonifier de façon plus significative le Social Security Act de 1935.

Un autre groupe, les Gray Panthers, se démarque sensiblement face aux précédents par sa composition sociale hétérogène et par l'absence d'une structure hiérarchique et bureaucratisée. En mettant l'accent sur le changement social global plutôt que sur le réajustement des politiques du système, en invoquant la nécessité de transcender les valeurs sociales et de niveler les structures, les Gray Panthers se situent en dehors du courant des groupes réformistes et modérés, tendance principale qui caractérise, de toute évidence, d'autres associations de citoyens âgés.

Les différences de classe sociale et de position idéologique entre les diverses associations ne les empêchent pas cependant de faire front commun dans plusieurs circonstances comme, par exemple, les coupures du gouvernement Reagan, en 1981. On ne peut cependant faire référence à un mouvement intégré et unifié des forces et des organisations engagées dans la défense des intérêts des personnes âgées. L'inscription des revendications dans le système politique demeure en partie morcelée et est fonction de la puissance des groupes qui les véhiculent et les soutiennent, tout comme de la position de ces groupes dans la stratification et le réseau des communications sociales. ${ }^{17}$ Conséquemment, l'appartenance de classe fait donc en sorte que les personnes âgées poursuivent des objectifs différents, ce qui exerce une contrainte dans la formation d'un véritable mouvement socio-politique unifié de personnes âgées.

\section{e) Vie associative et contraintes organisationnelles}

Les associations de personnes âgées se heurtent également aux contraintes inhérentes à la nature même des adhérents, du fait qu'ils sont vieillissants.

Malgré le fait que la participation associative s'accroisse depuis les dix dernières années, elle n'en demeure pas moins l'apanage d'une minorité généralement en bonne santé, plus éduquée que la moyenne, plus à l'aise financièrement et ayant pris l'habitude, au cours de la vie, de joindre les groupements volontaires. ${ }^{18,19,20}$ Les personnes âgées provenant de milieux vraiment défavorisés ne prennent habituellement pas l'initiative de s'intégrer à une association ou à un club, et encore moins d'en assurer le leadership. Elles sont plutôt encadrées par les institutions religieuses ou sociales, bien que, dans plusieurs cas, on vise 
à rendre la clientèle autonome. À cet effet, les associations ont de la difficulté, comme nombre d'autres groupes, à mobiliser des individus provenant de milieux défavorisés, bien qu'elles se disent quand même leur porte-parole.

Par ailleurs, Ward ${ }^{21}$ considère que la participation relativement faible des personnes âgées, toute proportion gardée, est troublante, en particulier si l'on considère que le groupe joue un rôle important comme mécanisme de soutien pour compenser les difficultés relationnelles (telles que : veuvage, isolement, etc.) subies au cours du vieillissement et en tant que mécanisme d'intégration pour combler la perte ou l'absence de rôle social.

Il y a plusieurs facettes à cette faible participation. II faut d'abord tenir compte des caractéristiques particulières de ce bassin de population. Certes, les personnes âgées disposent de plus de temps libre que la majorité de la population, mais le vieillissement impose son rythme et ses handicaps : autonomie fonctionnelle réduite, incidence de multipathologies, réduction de moyens financiers, insécurité, etc. "On travaille toujours avec des morts!" disait cette responsable d'un groupe québécois de personnes âgées. Compte tenu du taux de roulement, le recrutement est une préoccupation constante si l'on veut maintenir l'organisation en vie et sur une base permanente

De plus, l'implication active des participants est une condition importante de survie de l'organisme. Souvent le manque d'expérience et d'habileté en égard à la vie de groupe inhibe la personne âgée; il faut alors beaucoup de support et d'encouragement pour soutenir une implication active. Le manque d'aspirations, mais aussi une certaine mentalité de consommateur de services, peuvent également expliquer les forces d'inertie contre lesquelles il faut se battre pour maintenir le dynamisme d'une telle association.

Aux États-Unis, la majorité des participants ont entre 62 et 75 ans, sous-catégorie de la population âgée qu'on qualifie de "jeunes-vieux". Cette constatation nous amène à réduire, "statistiquement parlant ", le bassin de personnes susceptibles d'être mobilisées dans le cadre d'une association ou d'un groupement volontaire.

La faible participation peut aussi s'expliquer, en partie, par le fait que plusieurs ne veulent pas s'identifier à des groupes qui véhiculent une image de la vieillesse, souvent perçue, par la société, de façon péjorative. Ce serait alors, pour certains, "avouer" publiquement qu'on est un retraité. Dans une société fondée sur des valeurs de productivité et d'efficacité, on n'affiche pas toujours fièrement son âge ; de nombreuses études ont été réalisées dans le but d'explorer les caractéristiques de ce type d'identification. Entre autres, Cutler ${ }^{22}$ 
constate que seulement $38 \%$ des personnes âgées de son échantillonnage s'identifiaient sur cette base.

Dans cette même étude, certains s'identifiaient avec les jeunes alors que d'autres n'utilisaient pas du tout ce critère comme base de différenciation entre les gens. On a de plus constaté que les personnes vieillissantes qui ne s'identifient pas à leur groupe d'âge sont peu susceptibles de s'impliquer dans des associations ou des clubs. En corollaire, l'acceptation du statut de retraité est associée à l'acceptation des aspirations et revendications des groupes de retraités. ${ }^{23}$

D'autre part, les associations de personnes âgées rencontrent des problèmes qui sont propres à tout type de regroupement établi sur une base volontaire ${ }^{24}$ et, plus particulièrement, la difficulté, pour ce type de structure, de maintenir son existence dans le temps. De par sa nature, l'association volontaire repose sur la libre participation et sur l'énergie souvent bénévole de ses membres. Aussi, bien des facteurs peuvent détourner les participants tels les changements dans la vie personnelle (maladies, déménagements, etc.), de nouveaux intérêts ou des insatisfactions par rapport aux réalisations du groupe. Les considérations familiales et personnelles vont aussi avoir préséance sur celles qui sont dues à l'association et à sa survie.

Ce tour d'horizon des manifestations associatives aux États-Unis et des recherches qui y sont rattachées rend compte de préoccupations très présentes dans la société américaine et qu'on retrouve, à plusieurs égards, dans toutes les sociétés modernes. En examinant maintenant la situation en France, nous explorerons d'autres facettes du phénomène. Ainsi, une perspective d'analyse culturelle sera introduite, de même que l'analyse du rôle des politiques de la vieillesse sur la dynamique associative spécifique aux personnes âgées.

\section{France}

a) La structure française

D'après une recherche récente sur les mouvements associatifs de personnes âgées en France, Attias-Donfut et Rozenkier ${ }^{25}$ distinguent trois grands types: les clubs, les associations de retraités et celles de pré-retraités.

1. Les clubs de loisirs français. L'État français a joué un rôle significatif dans le développement massif de regroupements de personnes âgées, en particulier pour les clubs de loisirs. Voyant cette structure comme un instrument privilégié de participation sociale, le gouvernement a planifié, autour de 1965, la mise sur pied de cinq cents 
d'entre eux. Mais le développement de cette forme de vie sociale a dépassé les prévisions des planificateurs : plus de 2000 se sont constitués en l'espace de cinq ans ! Des estimations effectuées en 1983 portent ce nombre à environ 20000 . Il est à signaler que $74 \%$ d'entre eux sont implantés en milieu rural. Cet essor considérable est le résultat, d'une part, de la légitimation par l'État de "l'auto-organisation et de la représentation des personnes âgées " ${ }^{26}$ dans le cadre d'une politique de la vieillesse et, d'autre part, d'une grande mobilisation des personnes âgées elles-mêmes.

Le risque d'anomie et la recherche d'identité représenteraient aussi des facteurs explicatifs de cette mobilisation. Mais, dans une recherche de Collot ${ }^{27}$ portant sur les motivations et attentes des participants dans ces clubs de loisirs, la fuite de la solitude apparaît comme la motivation fondamentale de fréquentation. Certaines femmes expriment ainsi leur perception en se référant à : "La solitude !.. le réconfort, l'amitié, des trucs comme ça quoi !"; "Eh bien, vous savez, de rester chez soi entre ses quatre murs, c'est franchement pas gai." Ces besoins relationnels sous-jacents à la participation, tels qu'exprimés par les membres, ne diffèrent pas des besoins qui stimulent l'entrée de retraités américains dans un Senior club. Tel que le révèle une étude de Ward, ${ }^{28} 73 \%$ des répondants ont donné comme raison principale de fréquentation le contact avec des amis. Il existe donc un profond désir de socialisation chez la personne âgée et le club en constitue la voie d'accès.

2. Les associations de retraités. Les associations de retraités se rattachant à la vie professionnelle se sont principalement constituées en vue de défendre les intérêts de leurs membres et de créer des solidarités. Leurs activités sont largement diversifiées. Pour le président de la Fédération nationale des associations de retraités en France, Monsieur F.P. Mercereau, il est fondamental que le retraité organise sa nouvelle vie et fasse valoir ses droits dans des institutions qui lui sont propres. Contrairement aux discours qui interprètent le regroupement des personnes âgées comme une conséquence de leur marginalisation, il propose une vision positive de l'association entre personnes du même groupe d'âge. Ainsi, loin d'envisager ces regroupements comme des espaces pernicieux de ségrégation, Mercereau perçoit la vie associative comme un lieu d'un «mouvement intense d'échanges et de communications" entre personnes qui ont le même rythme de vie. "L'intergénération [...] à son heure et pas n'importe comment ! " ditil! ${ }^{29}$ Dans l'association qu'il préside (avec 415000 membres), les tâches s'organisent autour de divers pôles: activités physiques et culturelles, secteur d'action en faveur des personnes âgées, soutien à des groupes 
du milieu en difficulté, comme une minorité ethnique et des jeunes délinquants.

3. Les associations de pré-retraités. Les associations de pré-retraités, de constitution récente et encore peu nombreuses, sont issues de la crise de l'emploi qui frappe les travailleurs âgés, en France, et les incite à une prise de retraite hâtive. Initialement le domaine d'activités de ces groupes concernait surtout les loisirs et les activités culturelles. Mais, suite à un décret du gouvernement français par lequel les travailleurs doivent signer des conventions portant préjudice aux retraités, selon ces militants (notamment en regard des modalités financières), on a vu émerger un type plus revendicatif d'association de pré-retraités centrée sur la défense des intérêts et des droits sociaux. Les enjeux majeurs portent donc, actuellement, sur les conditions financières de la prise de retraite. Pour les militants, l'instauration de la pré-retraite risque de faire l'objet d'un nouveau conflit social.

\section{b) L'identité collective et ses conséquences}

1. Le pouvoir politique. La conscience d'appartenir à une nouvelle catégorie sociale désavantagée à plusieurs égards a conduit une minorité de retraités, par le biais de leurs associations volontaires, à militer comme nouveau pouvoir politique en faveur de la défense des droits des personnes âgées et de l'amélioration de leurs conditions de vie.

Certains chercheurs américains, comme nous l'avons vu précédemment, se sont interrogés sur les effets de "l'infiltration" de ce nouveau pouvoir gris au sein du système politique. On a ainsi constaté qu'il n'était pas menaçant mais forçait les autorités à prendre davantage en considération le sort de la population vieillissante et à formuler des mesures socio-économiques plus adéquates. II faut se rappeler, par ailleurs, que les besoins et aspirations des retraités se différencient selon leur classe sociale.

Le mouvement associatif des retraités, en France, ne semble pas porteur, non plus, d'une réelle contestation ou opposition au système social. «L'auto-organisation, la stratégie de négociation et de pression sont utilisées pour tenter de combler le décalage et assurer un certain niveau d'ajustement entre ce qui est conçu pour les retraités par les décideurs et ce qui est voulu par les retraités eux-mêmes. " ${ }^{30}$ De par sa nature revendicatrice, le mouvement associatif contemporain de personnes âgées peut, à juste titre, porter l'étiquette de mouvement réformiste modéré. 
En plus de rapports souvent conflictuels avec l'État, il faut également prendre en considération, dans la problématique de revendication de ces groupes, les rapports conflictuels avec les pouvoirs politiques régionaux et locaux. Finalement, mentionnons l'existence de conflits latents ou ouverts entre les groupements eux-mêmes, dans la mesure où les divers pouvoirs s'affrontent pour contrôler l'orientation sociale, politique et culturelle de la population âgée.

Les associations de retraités génèrent des activités économiques dont certaines échappent au système du marché conventionnel. Le troc de services se substitue, en partie, à la recherche de services sur le marché. Il se constitue donc alors une économie dite souterraine.

Ainsi, l'entraide, l'échange de biens et de services, le développement communautaire réalisés par les groupes constituent des formes d'intervention sociale qui contribuent de façon certaine au progrès social et à l'amélioration des conditions de vie de la population âgée, mais leurs retombées positives ne sont pas comptabilisées dans les comptes économiques nationaux. Ce phénomène n'exclut cependant pas le fait que les associations de personnes âgées deviennent, à plusieurs égards, des relais entre le réseau naturel, l'État et le secteur privé pour combler des besoins de diverses natures et assurer une ouverture communautaire aux personnes vieillissantes.

2. Culture du troisième âge. L'émergence d'une sous-culture du troisième âge a été envisagée comme une conséquence souhaitable, pour certains, ${ }^{31}$ de l'identification collective, qui permettrait une meilleure adaptation à l'environnement social, l'individu s'y référant alors comme réalité sociale plutôt qu'à une culture plus générale envers laquelle il est plus difficile de s'intégrer et de s'ajuster, compte tenu de ses exigences. Dans certaines communautés de retraités où les conditions maximales de développement d'une sous-culture sont présentes (proximité géographique, interaction sociale soutenue avec les pairs, etc.), on a constaté que les personnes âgées vivant en milieu homogène avaient une meilleure image de soi. Elles se sentaient cependant moins utiles que les retraités vivant en milieu hétérogène ${ }^{32}$ et n'étaient pas plus actives socialement que ces derniers. Contrairement à une orientation activiste et politique, ces communautés de retraite véhiculent des moủeles de «désengagement collectif ». L'émergence d'une sous-culture représente un phénomène marginal spécifique à certains milieux de vie et «on ne peut s'attendre à ce qu'il débouche en une forme de sous-culture particulière à toute la strate de personnes âgées". ${ }^{33}$

À l'opposé du désengagement collectif de certaines communautés de retraités, la vie associative véhicule des modèles culturels qu'on 
pourrait qualifier, de façon générale, d'idéologie d'un âge actif et participant (activisme).

Par exemple, dans une étude portant sur la vie associative des retraités de l'enseignement, Meunier ${ }^{34}$ distingue trois modes de participation :

- Action-organisation : il s'agit d'un mode de participation qui reflète fidèlement la structuration du temps pendant la vie professionnelle; ce type de retraité prend des responsabilités au sein des associations; il a souvent le sentiment de manquer de temps.

- Participation-liberté : pour cette catégorie, la liberté d'action et le temps libre sont des dimensions valorisées ; "c'est l'envers du temps contraint, de l'espace assigné ".

- Invention du quotidien : ce mode de participation est axé avant tout sur la réalisation de soi, la créativité.

Ces exemples de participation associative rendent compte de modèles culturels dynamiques et créateurs, particuliers à une catégorie de professionnels des classes moyenne ou supérieure et qui embrassent l'idéologie d'un troisième âge actif et participant.

Par ailleurs, dans une recherche portant sur les modèles culturels véhiculés par la presse du troisième âge à propos de la vie associative dans les clubs de loisirs, Mercier ${ }^{35}$ identifie trois modèles de relations :

- le modèle de vie communautaire privilégie l'amitié et la solidarité dans un univers associatif serein et harmonieux;

- le modèle libertaire présente le club comme un lieu de liberté de parole ;

- le modèle activiste met l'emphase sur le dynamisme de la personne âgée; celle-ci devant conquérir sa place dans la société et se prendre en charge.

Il est souligné que le discours de ces clubs de loisirs est "séduisant et plein, à l'opposé d'un discours analytique et critique" ${ }^{36}$ qui marquerait l'écart entre les aspirations des personnes âgées et leur quotidien. Le club est présenté ici comme un lieu idéalisé semblable à la communauté villageoise d'autrefois, et l'action y est survalorisée aux dépens de la réflexion et du travail psychique. Ce discours, selon l'auteur, produit des images leurrantes et assure "une mise à distance des images mortifères qui accompagnent le vieillissement $" .{ }^{37}$

Cette position reflète, en partie, une critique de l'activisme comme modèle de bien vieillir à la retraite. II s'agit d'un modèle où l'idéal type est une personne âgée, homme ou femme, au regard "jeune", impliquée socialement et développant son potentiel dans des activités 
physiques, culturelles et éducatives tout à la fois. La critique de l'activisme s'appuie notamment sur l'idée qu'il est inadéquat de transposer des normes de comportement spécifique à la vie adulte alors qu'on s'adresse à une population vieillissante, graduellement en perte d'autonomie et de réserve d'énerge.

Malgré les critiques qui lui sont adressées, l'idéologie d'un troisième âge actif et dynamique constitue un modèle fort présent dans la société actuelle et anime les milieux associatifs depuis les années 1960. Cette façon de voir, encouragée par les politiques de la vieillesse, résulte des changements qui ont marqué les sociétés dans les dernières décennies, en particulier : l'émergence d'une nouvelle classe moyenne, la valorisation du loisir, l'accession à une retraite de plus en plus hâtive, donc des retraités plus jeunes et énergiques.

\section{c) Impact des politiques sur la vieillesse}

sur la vie associative

Dans une étude récente, Guillemard ${ }^{38}$ propose une grille de lecture de la gestion sociale de la vieillesse qui met en rapport le contexte social avec les modes successifs de prise en charge des personnes âgées. Ceci permet de mieux comprendre les forces sociales qui sont présentes et qui agissent dans leur dynamique associative. Les éléments principaux de la grille de lecture sont identifiés dans le tableau suivant.

Bien que cette étude s'appuie sur des données provenant de la société française, les trois périodes identifiées reflètent, de façon générale, les orientations qui ont marqué la vie associative des personnes âgées dans plusieurs autres démocraties occidentales.

Durant la première période, "l'image dissuasive de la vieillesse comme indigence $[. .$.$] a eu une valeur exemplaire et a contribué, tout$ au long du XIX ${ }^{\mathrm{e}}$ siècle, à la formation morale des adultes et des enfants des classes populaires, en les incitant à la prévoyance et à l'économie ". ${ }^{39}$ C'est dans la seconde période, caractérisée par l'idéologie d'un troisième âge actif et participant, que les groupes et associations de personnes âgées prennent leur essor, qui fut légitimé par les discours de l'État et facilité par l'amélioration des conditions de vie de la population âgée. Par opposition à la première période, il s'agit d'une nouvelle logique sociale à l'égard de la vieillesse qui opère un changement profond des mentalités. Au cours de la seconde période, le rapport à l'avenir est fondé sur «des stratégies de prévision et non sur la simple prévoyance». ${ }^{40}$ La stimulation à la consommation, l'émergence de nouvelles couches moyennes salariées porteuses 


\section{TABLEAU}

Contexte social et modes successifs de prise en charge des personnes âgées (modèle français)

\begin{tabular}{|c|c|c|c|}
\hline Contexte & 1945 à 1960 & 1960 à 1970 & 1970 à nos jours \\
\hline $\begin{array}{l}\text { Modes dominants } \\
\text { de gestion sociale }\end{array}$ & $\begin{array}{l}\text {-Pension de vieillesse } \\
\text {-Assistance publique }\end{array}$ & $\begin{array}{l}\text { Politique globale sur la vieillesse } \\
\text { et mise en place d'un réseau } \\
\text { d'équipements et de services } \\
\text { spécialisés }\end{array}$ & $\begin{array}{l}\text {-Consolidation de la politique } \\
\text { sur la vieillesse } \\
\text {-Politiques de pré-retraite }\end{array}$ \\
\hline Enjeux dominants & $\begin{array}{l}\text { Conditions d'existence de la } \\
\text { population âgée (niveau de vie) }\end{array}$ & Mode de vie et insertion sociale & Niveau de vie et âge de la retraite \\
\hline $\begin{array}{l}\text { Représentation } \\
\text { sociale } \\
\text { de la vieillesse }\end{array}$ & $\begin{array}{l}\text { - Nouvelle perception des con- } \\
\text { ditions de vie des vieillards } \\
\text {-Vieillesse associée à la misère, } \\
\text { à la déchéance } \\
\text {-Référents sociaux: personnes } \\
\text { âgées des classes populaires }\end{array}$ & $\begin{array}{l}\text {-Pour une vieillesse active, } \\
\text { fondée sur l'autonomie } \\
\text {-Référents sociaux: nouvelles } \\
\text { couches moyennes salariées }\end{array}$ & $\begin{array}{l}\text {-Érosion du droit au travail } \\
\text {-Dévalorisation et marginali- } \\
\text { sation des travailleurs âgés } \\
\text {-Nouvelle définition de la vieil- } \\
\text { lesse qui commence plus tôt } \\
\text { dans le cycle de vie }\end{array}$ \\
\hline
\end{tabular}


d'aspirations et de ressources concourent à la redéfinition des représentations sociales de la vieillesse et à l'orientation de son devenir. Conséquemment, une large part des activités associatives s'organise en fonction du loisir. Cette fonction récréative est alimentée et orientée, à plusieurs égards, par les incitations multiples à la consommation. Amorcée dans la seconde période, cette fonction récréative se poursuit durant la suivante; elle se spécialise, se raffine et intègre les modes les plus récentes: danse aérobique, gymnastique, voyages, etc.

Cette perspective récréative n'écarte cependant pas le souci d'améliorer les conditions de vie de la population âgée; nombre d'associations s'y intéressent et certaines en font leur raison d'être. Durant la troisième période, où dominent les débats sur le niveau de vie et l'âge de la retraite, ce qui coïncide avec des années de décroissance économique, l'évacuation précoce des travailleurs âgés devient le cheval de bataille de nouveaux types d'associations: celles de préretraités. La fonction revendicatrice se raffermit et les associations de personnes âgées font définitivement leur entrée comme groupes de pression dans le système politique.

Cette lecture sociologique démontre que le développement associatif est indissociable de l'évolution des politiques sociales, de la situation économique et de la configuration des populations.

Nous terminerons la revue des publications en décrivant sommairement la situation au Québec.

\section{Québec}

Depuis les années 1960, il s'érige au Québec une structure associative solide qui reçoit, dans plusieurs cas, le support des gouvernements provincial et fédéral. Par exemple, le gouvernement fédéral a fortement stimulé les retraités à s'impliquer dans la vie associative lorsqu'il a introduit le programme de subventions "Nouveaux Horizons", en 1972, par l'entremise du Ministère de la santé nationale et du bien-être social.

Aujourd'hui, des groupes actifs, dans tous les coins du Québec, témoignent de la vitalité d'hommes et de femmes retraités qui se rencontrent pour partager des intérêts et préoccupations communes, rompre leur solitude et améliorer leurs conditions de vie. Ces aînés se regroupent sur la base du type d'employeur ou selon l'appartenance professionnelle (par exemple: association des fonctionnaires à la retraite), sur la base des lieux de résidence (association des résidents en H.L.M.), ou du quartier (groupes issus de C.L.S.C. ou amorcés par des congrégations religieuses). La nationalité ainsi que la religion constituent 
également des facteurs de regroupement : le Chinese Old Age Center, l'Association des vieillards grecs, la Vie Montante en sont des exemples. Il en est ainsi de champs d'intérêt précis comme le jardinage, la musique ou l'histoire, sous l'initiative de retraités enthousiasmés. On relève aussi la présence de regroupements d'associations, telle l'Association internationale des aînés francophones. De plus, le Forum des citoyens âgés, issu du milieu anglophone, en 1965, constitue un lieu d'échanges et de concertation pour les retraités de la région de Montréal.

Les Clubs de l'Âge d'or représentent, par ailleurs, le type de regroupement le plus visible. Avec le support du Conseil des oeuvres et du bien-être du Québec (C.O.B.E.Q.), le mouvement de l'Âge d'or voit ses débuts vers $1966 .{ }^{41}$ En 1969, on assiste à la création du premier Conseil de l'Âge d'or, et, en 1970, naît la F.A.D.O.Q. : Fédération de l'Âge d'or du Québec, responsable de l'orientation du mouvement, et qui se définit comme la représentante de cette population. Ce mouvement lutte contre ce qu'il appelle les " $4-i$ ", à savoir : insécurité, isolement, inactivité, et inutilité. On estime à environ 160000 le nombre de personnes âgées de 55 ans et plus qui participent aux activités des Clubs de l'Âge d'or. Celles-ci se sont aujourd'hui diversifiées et couvrent autant le loisir que l'entraide communautaire et le développement culturel. Avec les années, l'élargissement de leurs fonctions a contribué à modifier sensiblement l'image que la population s'en est faite comme étant des lieux réservés aux joueurs et joueuses de cartes, bien que cette activité demeure très populaire.

Plus récemment, en mai 1980, est née l'Association québécoise pour la défense des droits des retraités et des pré-retraités (A.Q.D.R.), qui se définit principalement comme un groupe de conscientisation et de revendication des droits des retraités. Elle rejoindrait environ 9500 membres. Elle s'est fait connaître, localement et auprès des gouvernements, en publiant des manifestes sur les conditions de vie de la population âgée et en acheminant des revendications précises aux autorités dans les domaines du revenu, du logement, du maintien à domicile et du transport adapté.

En décembre 1981 naît la Fédération québécoise des retraités en résidences et centres d'accueil (F.Q.R.R.C.A.) dont le nom sera changé, par la suite, en Fédération des aînés du Québec. Elle se veut un regroupement socio-éducatif communautaire, visant l'éducation et l'information des retraités québécois. Encore jeune, elle tente de se tailler une place, à mi-chemin entre la F.A.D.O.Q. et l'A.Q.D.R.

Cette description n'épuise pas la richesse des manifestations associatives au Québec. Dernièrement, les leaders d'associations, dont 
la nouvelle présidente de la F.A.D.O.Q. et le président de la Fédération des aînés du Québec, ont participé à la création d'une commission permanente des aînés, front commun pour donner un véritable droit de parole aux personnes âgées et consolider ainsi l'action revendicatrice en leur faveur. ${ }^{42}$

Bien qu'au Québec aucune recherche n'aborde de façon spécifique le phénomène associatif propre aux personnes âgées, les propos recueillis dans les journaux, ainsi que des rencontres avec des participants âgés et des animateurs de l'Âge d'or, laissent supposer que la dynamique associative participe ici d'un même processus de différenciation sociale et qu'elle n'est pas exempte de rapports conflictuels avec les autorités locales ou entre associations elles-mêmes, soucieuses de monopoliser le plus grand nombre d'adhérents. L'implication active des participants âgés demeure aussi une préoccupation constante des leaders.

\section{Conclusion}

Suite à cette revue de la littérature, on constate que les chercheurs commencent, depuis quelques années, à ausculter la vie associative des personnes âgées sous divers angles. Celles-ci de leur côté investissent de plus en plus dans diverses formes de regroupements. $\grave{A}$ la lumière des données empiriques et des discours, ce phénomène peut être circonscrit à partir de quatre fonctions qui correspondent aux dimensions expressives ou instrumentales de l'association volontaire :

- expressive: fonction psycho-sociale, fonction récréative ;

- instrumentale: fonction de développement communautaire, fonction de revendication socio-politique.

Nous donnons ici une brève description de chacune d'elles.

Pour la personne âgée, l'appartenance associative constitue une ressource actuelle ou potentielle qui lui permet de satisfaire des besoins de socialisation, d'appartenance, de sécurité et de réalisation de soi. Cette ressource est utilisée ou transformée, selon les compétences et les aspirations de la personne, pour structurer sa vie à la retraite.

La société de consommation et de loisirs s'est "infiltrée» dans la vie associative et offre ses produits à la personne âgée : voyages, activités physiques, culturelles, artisanat, etc. Tout en lui assurant une ouverture sur le monde, la fonction récréative met en quelque sorte la vieillesse en latence et exorcise ses misères. 
Plusieurs associations ont développé des actions dans le but de s'impliquer dans leur milieu immédiat. Ces réalisations discrètes $s^{\prime}$ insèrent dans le développement communautaire, de façon certaine mais encore peu connue. Il s'agit d'une fonction de relais entre les technocraties et le milieu naturel.

Par le biais de leurs associations, les personnes âgées se sont graduellement données une voix officielle qui ne peut plus être écartée de la conscience politique, d'autant plus qu'elle est l'écho d'une tranche de population qui s'accroît. Ce porte-parole rend compte des frustrations économiques que vit cette population. Les militants réclament de meilleures conditions de vie mais aussi un respect de l'individu vieillissant. C'est une fonction que leurs associations sont appelées à remplir, de plus en plus.

Malgré des systèmes politiques et des cultures différentes, les associations de personnes âgées se sont taillé une place dans la structure de la vie sociale contemporaine. Ces groupes sont perméables aux dynamismes sociaux qui animent la société moderne comme tout autre regroupement volontaire non constitué sur la base de l'âge.

Les fonctions de la vie associative actuelle illustrent bien la volonté collective des retraités d'agir sur le plan socio-communautaire et politique dans le but d'améliorer leurs conditions de vie. Par ailleurs, les activités offertes permettent à la personne âgée de s'actualiser et de participer aux courants de la vie socio-culturelle. Loin de se marginaliser, la population âgée s'inscrit dans le processus de la modernisation par différentes voies, dont celle de la vie associative.

Il est fort souhaitable que les intervenants sociaux établissent une collaboration encore plus étroite avec les groupes et leurs leaders dans une perspective d'échange et de complémentarité. Chaque partenaire pourra ainsi enrichir ses pratiques sociales et contribuer au développement de canaux de communication plus féconds entre les technocraties et la population âgée.

\section{Références bibliographiques}

1 Rosenmayer, L., "Les étapes de la vie», Communications, no 37, 1983 : 89-104.

2 Encyclopedia of Associations, Détroit (Mi.), Gale Research Co., publiée depuis 1959.

3 Dıon, L., Société politique : la vie des groupes, tome I, Québec, Les Presses de l'Université Laval, 1971, p. 203.

4 Meister, A., Vers une sociologie des associations, Paris, Éditions Économie et humanisme, 1972. 
5 KLeEMEIER, R.W., Aging and Leisure. A Research Perspective into the Meaningful Use of Time, New York, Oxford University Press, 1961.

"LESSARD, J., "Les bénévoles, une force démocratique", allocution prononcée au congrès de l'Association du diabète du Québec, Montréal, le 13 octobre 1984.

7 CARLIE, M.K., "The politics of age : interest group or social movement", The Gerontologist, vol. 9, no 3, 1969: 259-263.

8 PRATT, H.J., The Gray Lobby, Chicago, University of Chicago Press, 1976.

9 Ibid.

10 Harris, L. et al. (éds), The Myth and Reality of Aging in America. A Study for the National Council of Aging Inc., Washington, 1975.

11 Attias-Donfut, C., "Les clubs pour personnes âgées : naissance d'une nouvelle force sociale", Cahiers de l'animation, no 9, 1975, p. 28.

12 Rose, A.M. et W.A. Peterson (éds), Older People and their Social World, Philadelphie (Penn.), F.A. Davis, 1965.

13 Pratt, H.J., op. cit.

14 Hartoyan, R.A., Interest Groups and Aging Policy, dans: Robert Hudson (éd.), Aging in Politics: Process and Policy, Springfield (III.), Charles C. Thomas, 1981, p. 80.

15 Levasseur, R., "Le phénomène associatif ", Cahiers de l'animation, n 39, 1983, p. 36.

16 Pratt, H.J., National Interest Groups Among the Elderly: Consolidation and Constraint, dans: W.P. Browne et L.K. Olson (éds), Aging and Public Policy, the Politics of Growing Old in America, Westport (Conn.), Greenwood Press, 1983: 145-180.

17 Poujol, G., "La dynamique sociale des associations», Cahiers de l'animation, no $39,1983: 57-79$.

18 TiвBıтTS, C. (éd.), Handbook of Social Gerontology, Societal Aspects of Aging, Chicago (III.), University of Chicago Press, 1960. Voir, en particulier, le huitième chapitre: A.M. Rose, The Impact of Aging on Voluntary Associations: 666-697.

19 Pratt, H.J., 1983, op. cit.

20 Lowenthal, M.F. et B. Robinson, Social Networks and Isolation, dans: R. Binstock et E. ShanAs (éds), Handbook of Aging and the Social Sciences, New York, Van Nostrand, 1976: 432-456.

21 WARD, R.A., "The meaning of voluntary association participation to older people", Journal of Gerontology, vol. 34, no 3, 1979: 438-445.

22 CUtLeR, S.J., "Aging and voluntary association participation", Journal of Gerontology, vol. 32, no 4, 1977 : 470-479.

23 Browne, W.P. et K.L. Olson, op. cit.

24 TibBitTs, C., op. cit.

25 Attias-Donfut, $C$. et $A$. Rozenkier, "Des clubs du troisième âge aux mouvements associatifs: une dynamisation sociale des personnes âgées", Gérontologie et société, no 26, $1983:$ 89-100.

26 Attias-Donfut, C. et A. Rozenkier, op. cit., p. 93. 
27 Collot, C., Les clubs pour personnes âgées en France, motivations et attentes des participants, Paris, Centre international de gérontologie sociale, 1975, p. 38.

28 WARD, R.A., op. cit.

29 Mercereau, F.P., "La Fédération nationale des associations de retraités", Gérontologie et société, no 26, 1983, p. 112.

30 Attias-Donfut, C. et A. Rozenkier, op. cit., p. 94.

31 Rose, A.M. et W.A. Peterson, op. cit.

32 Longino, C.F., K.A. McClelland et W.A. Peterson, "The aged subculture hypothesis: social integration, gerontophilia and self-conception", Journal of Gerontology, vol. 35, no 5, 1980 : 758-767.

33 Massé, J. et M. Brault, "Sociétés, vieillissement et stratification des âges", Sociologie et sociétés, vol. 16, no 2, 1984, p. 11.

34 MeUnier, C., "Participation associative et retraite», Gérontologie et société, no 26, 1983, p. 55.

35 MerCter, J.-Y., "Les échos du troisième âge ", Gérontologie et société, no 17, $1981: 91-97$.

36 Mercier, J.-Y., op. cit., p. 96.

37 Id., p. 97.

38 Guillemard, A.M., "La production des politiques de la vieillesse», Communications, no 37, 1983 : 105-124.

39 Id. , p. 118.

40 Ibid.

${ }^{41}$ Données recueillies lors d'une rencontre avec un intervenant du Conseil régional de l'Âge d'or, région de Québec.

42 Ibid. 